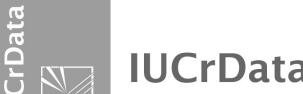

ISSN 2414-3146

Received 19 November 2016

Accepted 27 November 2016

Edited by J. Simpson, University of Otago, New Zealand

Keywords: crystal structure; low temperature; polymeric lead thiourea salt.

CCDC reference: 1519430

Structural data: full structural data are available from iucrdata.iucr.org

\section{Poly[di- $\mu_{2}$-acetato- $\kappa^{4} O: O^{\prime}-\mu_{3}$-thiourea- $\kappa^{3} S: S: S-$ lead(II)]: a redetermination}

\author{
Hafid Zouihri,* Mbark Ait Mouha, Bouchra Ba Mohamed, Khalid Yamni and Najib \\ Tijani
}

Laboratoire de Chimie des Matériaux et Biotechnologie des Produits Naturels, E.Ma.Me.P.S, Université Moulay Ismail, Faculté des Sciences, Meknès, Morocco. *Correspondence e-mail: hafid.zouihri@gmail.com

The structure of the title polymeric lead(II) thiourea complex, $\left[\mathrm{Pb}\left(\mathrm{CH}_{3} \mathrm{O}\right)_{2}\left\{\mathrm{SC}\left(\mathrm{NH}_{2}\right)_{2}\right\}\right]_{n}$, has been redetermined at significantly higher precision using diffractometer data at $100 \mathrm{~K}$. The previous determination used data obtained from multiple-film integrated Weissenberg photographs [Nardelli et al. (1960). Acta Cryst. 13, 898-904]. The main difference between the two models is in the precision of the bond lengths, angles and cell parameters. In the crystal, the eight-coordinate $\mathrm{Pb}^{\mathrm{II}}$ atom is chelated by two carboxylate groups and bridged by three $\mathrm{S}$ atoms from thiourea ligands. The coordination sphere is completed by an $\mathrm{O}$ atom from a third carboxylate group, the second $\mathrm{O}$ atom of which binds to a neighbouring $\mathrm{Pb}^{\mathrm{II}}$ atom, forming a polymeric chain that runs the $a$ axis. Two of these chains are related by centres of symmetry. Intermolecular hydrogen bonds connect neighbouring chains to one another, generating a three-dimensional network.
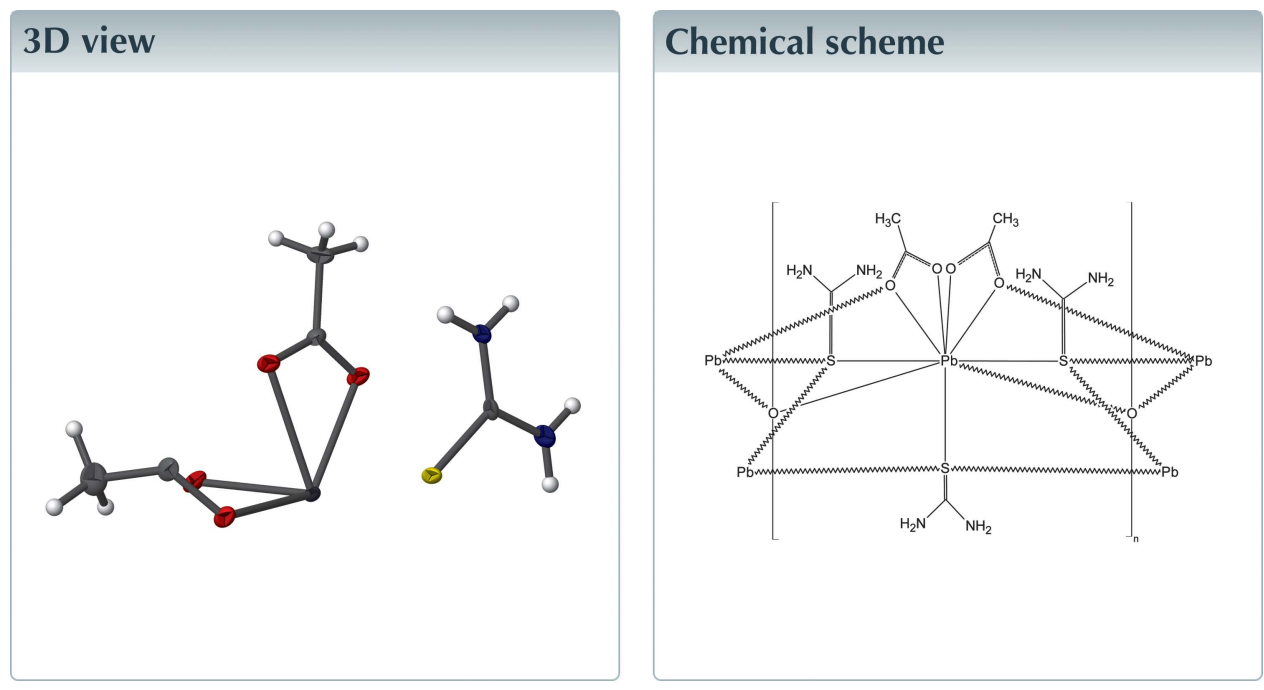

\section{Structure description}

In the polymeric complex, $\left[\mathrm{Pb}\left(\mathrm{CH}_{3} \mathrm{O}\right)_{2}\left\{\mathrm{SC}\left(\mathrm{NH}_{2}\right)_{2}\right\}\right]_{n}$, (Fig. 1) an infinite one-dimensional polymeric chain propagates along the $a$ axis (Fig. 2) with the $\mathrm{Pb}^{\mathrm{II}}$ ions chelated by the $\mathrm{O}$ atoms of two carboxylate groups and bridged by three $\mathrm{S}$ atoms from thiourea ligands related to a neighbouring $\mathrm{Pb}$ atom by a centre of symmetry. The eightfold coordination is completed by an oxygen atom from a third carboxylate group (Fig. 2). The $\mathrm{Pb}-\mathrm{O}$ bond lengths range from 2.483 (2) to 2.626 (2) $\AA$, while the two unique $\mathrm{Pb}-\mathrm{S}$ bonds are 3.0701 (9) and 3.1121 (9) $\AA$, respectively. The $\mathrm{Pb}$ atom is displaced out of the leastsquares planes of the carboxylate groups $(A=\mathrm{O} 1 / \mathrm{C} 1 / \mathrm{O} 2)$ and $(B=\mathrm{O} 3 / \mathrm{C} 3 / \mathrm{O} 4)$ by 0.0038 (2) and 0.0078 (3) $\AA$, respectively. The dihedral angles between the mean planes of 
Table 1

Hydrogen-bond geometry $\left(\AA,^{\circ}\right)$.

\begin{tabular}{lllll}
\hline$D-\mathrm{H} \cdots A$ & $D-\mathrm{H}$ & $\mathrm{H} \cdots A$ & $D \cdots A$ & $D-\mathrm{H} \cdots A$ \\
\hline $\mathrm{N} 2-\mathrm{H} 2 N B \cdots \mathrm{O} 4^{\mathrm{i}}$ & 0.86 & 1.99 & $2.836(4)$ & 169 \\
$\mathrm{~N} 2-\mathrm{H} 2 N A \cdots \mathrm{O} 1^{\mathrm{ii}}$ & 0.86 & 2.04 & $2.883(4)$ & 168 \\
$\mathrm{~N} 1-\mathrm{H} 1 N A \cdots 2^{\mathrm{iii}}$ & 0.86 & 2.26 & $3.021(4)$ & 148 \\
$\mathrm{~N} 1-\mathrm{H} 1 N B \cdots \mathrm{O} 3^{\text {iv }}$ & 0.86 & 2.48 & $3.311(4)$ & 163 \\
$\mathrm{C} 4-\mathrm{H} 4 A \cdots \mathrm{O} 4$ & 0.96 & 2.51 & $3.225(5)$ & 131 \\
\hline
\end{tabular}

Symmetry codes: (i) $x+\frac{1}{2},-y+\frac{1}{2}, z-\frac{1}{2}$; (ii) $x+1, y$, ; (iii) $x-\frac{1}{2},-y+\frac{1}{2}, z-\frac{1}{2}$; (iv) $-x+2,-y,-z$.

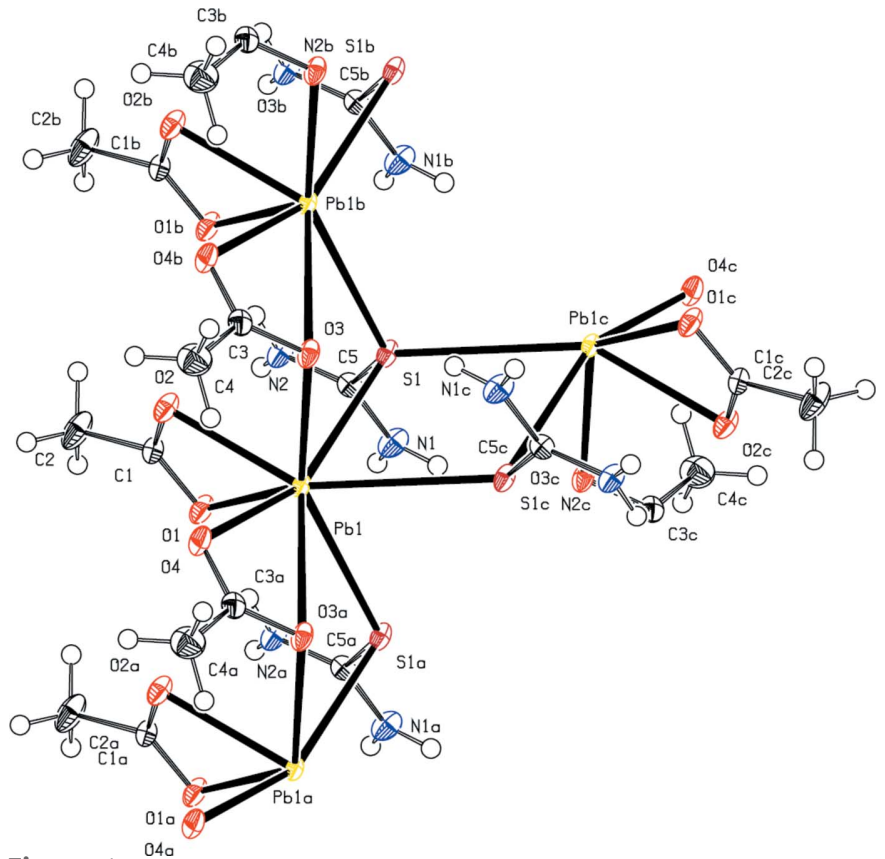

Figure 1

The polymeric chain in the structure of the title compound, showing the atom-labeling scheme. Displacement ellipsoids are drawn at the $30 \%$ probability level. $\mathrm{H}$ atoms are represented as small spheres of arbitrary radii. Atom labels with the suffix a, b and $\mathrm{c}$ are related to those with no suffix by the symmetry operations (i), (ii) and (iii) in Table 1 .

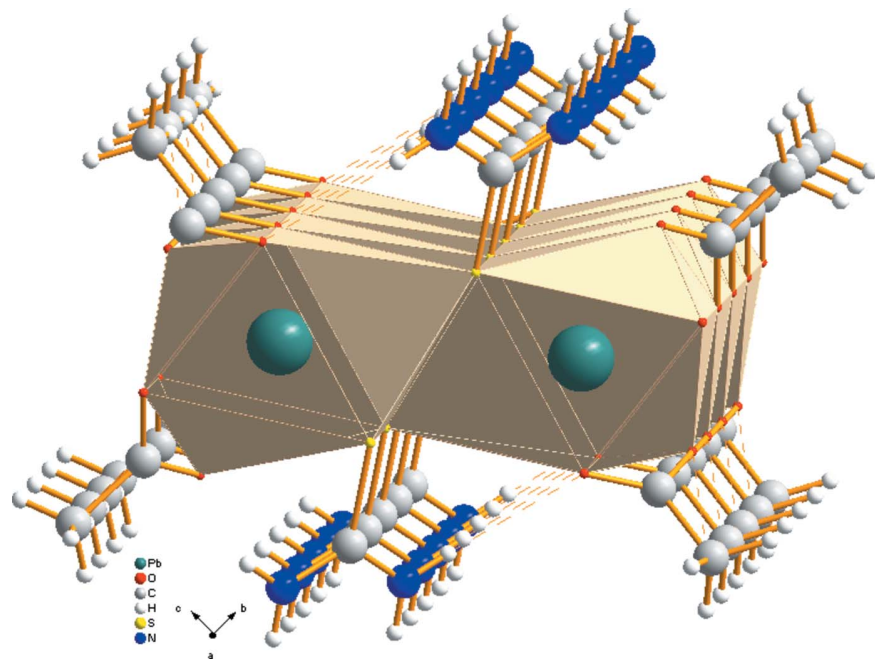

Figure 2

View of a polymeric chain of in the structure of the title compound. For clarity, the $\left[\mathrm{PbO}_{5} \mathrm{~S}_{3}\right]$ units are shown as polyhedra, the atoms of the organic ligands are represented as spheres of uniform size selected for each atom type. $\mathrm{N}-\mathrm{H} \cdots \mathrm{O}$ and $\mathrm{C}-\mathrm{H} \cdots \mathrm{O}$ hydrogen bonds within the chain are shown as dashed lines.
Table 2

Experimental details.

Crystal data

Chemical formula

$M_{\mathrm{r}}$

Crystal system, space group

Temperature (K)

$a, b, c(\AA)$

$\beta\left({ }^{\circ}\right)$

$V\left(\AA^{3}\right)$

$Z$

Radiation type

$\mu\left(\mathrm{mm}^{-1}\right)$

Crystal size (mm)

$\left[\mathrm{Pb}\left(\mathrm{C}_{2} \mathrm{H}_{3} \mathrm{O}_{2}\right)_{2}\left(\mathrm{CH}_{4} \mathrm{~N}_{2} \mathrm{~S}\right)\right]$

401.40

Monoclinic, $P 2_{1} / n$

100

4.4865 (2), 15.7001 (5), 13.6313 (5)

$91.481(2)$

$959.85(6)$

Mo $K \alpha$

17.78

Data collection

Diffractometer

Absorption correction

$T_{\min }, T_{\max }$

No. of measured, independent and observed $[I>2 \sigma(I)]$ reflections

$R_{\text {int }}$

$(\sin \theta / \lambda)_{\max }\left(\AA^{-1}\right)$

$0.32 \times 0.27 \times 0.13$

Bruker APEXII CCD detector Multi-scan ( $S A D A B S$; Bruker, 2009)

$0.005,0.099$

$13626,1893,1824$

0.036

0.617

\section{Refinement}

$R\left[F^{2}>2 \sigma\left(F^{2}\right)\right], w R\left(F^{2}\right), S \quad 0.015,0.037,1.08$

No. of reflections

No. of parameters

No. of restraints

$\mathrm{H}$-atom treatment

$\Delta \rho_{\max }, \Delta \rho_{\min }\left(\mathrm{e} \AA^{-3}\right)$

$\mathrm{H}$-atom parameters constrained $0.88,-1.22$

Computer programs: APEX2 and SAINT (Bruker, 2009), SHELXT (Sheldrick, 2015a), SHELXL2014 (Sheldrick, 2015b), PLATON (Spek, 2009) and publCIF (Westrip, 2010).

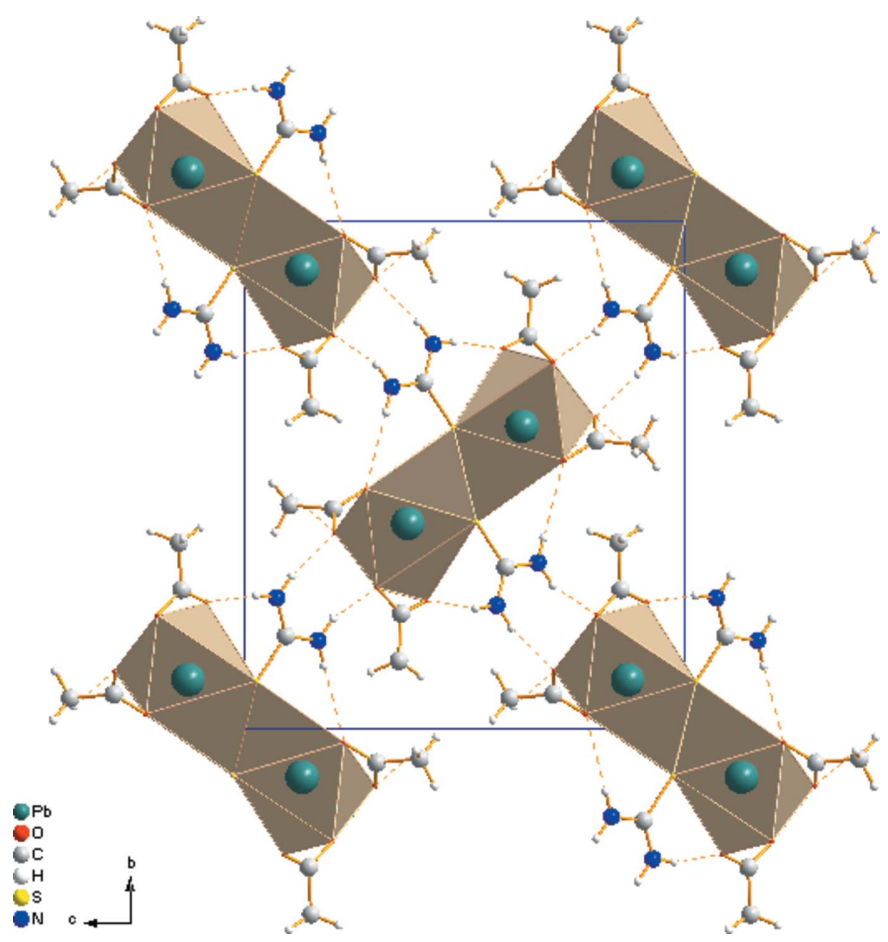

Figure 3

The crystal packing of the title compound viewed along the $a$ axis. N$\mathrm{H}$... O hydrogen bonds are shown as dashed lines (see Table 1 for details). 
thiourea ligands (S1/C5/N1/N2) and the carboxylate groups $A$ and $B$ are 6.93 (18) and $64.37(19)^{\circ}$, respectively.

In the crystal, $\mathrm{H}$ atoms are involved in inter-chain $\mathrm{N}-$ $\mathrm{H} \cdots \mathrm{O}$ and intra-chain $\mathrm{N}-\mathrm{H} \cdots \mathrm{O}$ and $\mathrm{C}-\mathrm{H} \cdots \mathrm{O}$ hydrogen bonds. These link the polymeric chains and stabilize the crystal structure, forming a three-dimensional network (Fig. 3, Table 1).

\section{Synthesis and crystallization}

The title compound was obtained from a mixture of (diaminomethylidene)sulfonium chloride/thiourea

(Zouihri, 2012) and lead acetate in a molar ratio of $1: 1$ in ethanol. The mixture was then left for slow evaporation and colourless crystals formed after four days.

\section{Refinement}

Crystal data, data collection and structure refinement details are summarized in Table 1 . One reflection (011) with $F_{\mathrm{o}}<<<$ $F_{\mathrm{c}}$, likely to have been affected by the beamstop, was omitted from the final refinement.

\section{References}

Bruker (2009). APEX2, SAINT and SADABS. Bruker AXS Inc., Madison, Wisconsin, USA.

Nardelli, M., Fava, G. \& Branchi, G. (1960). Acta Cryst. 13, 898-904. Sheldrick, G. M. (2015a). Acta Cryst. A71, 3-8. Sheldrick, G. M. (2015b). Acta Cryst. C71, 3-8. Spek, A. L. (2009). Acta Cryst. D65, 148-155. Westrip, S. P. (2010). J. Appl. Cryst. 43, 920-925.

Zouihri, H. (2012). Acta Cryst. E68, o257. 


\section{full crystallographic data}

IUCrData (2016). 1, x161892［https://doi.org/10.1107/S2414314616018927]

Poly[di- $\mu_{2}$-acetato- $\kappa^{4} O: O^{\prime}-\mu_{3}$-thiourea- $\left.\kappa^{3} S: S: S-l e a d(I I)\right]:$ a redetermination

Hafid Zouihri, Mbark Ait Mouha, Bouchra Ba Mohamed, Khalid Yamni and Najib Tijani

Poly[di- $\mu_{2}$-acetato- $\kappa^{4} O: O^{\prime}-\mu_{3}$-thiourea- $\kappa^{3} S: S: S$-lead(II)]

Crystal data

$\left[\mathrm{Pb}\left(\mathrm{C}_{2} \mathrm{H}_{3} \mathrm{O}_{2}\right)_{2}\left(\mathrm{CH}_{4} \mathrm{~N}_{2} \mathrm{~S}\right)\right]$

$M_{r}=401.40$

Monoclinic, $P 2_{1} / n$

$a=4.4865(2) \AA$

$b=15.7001(5) \AA$

$c=13.6313(5) \AA$

$\beta=91.481(2)^{\circ}$

$V=959.85(6) \AA^{3}$

$Z=4$

$F(000)=736$

$D_{\mathrm{x}}=2.778 \mathrm{Mg} \mathrm{m}^{-3}$

Mo $K \alpha$ radiation, $\lambda=0.71073 \AA$

Cell parameters from 214 reflections

$\theta=3.1-26.4^{\circ}$

$\mu=17.78 \mathrm{~mm}^{-1}$

$T=100 \mathrm{~K}$

Prism, colourless

$0.32 \times 0.27 \times 0.13 \mathrm{~mm}$

\section{Data collection}

Bruker APEXII CCD detector

diffractometer

Radiation source: fine-focus sealed tube

Graphite monochromator

$\omega$ and $\varphi$ scans

Absorption correction: multi-scan

(SADABS; Bruker, 2009)

$T_{\min }=0.005, T_{\max }=0.099$

13626 measured reflections

1893 independent reflections

1824 reflections with $I>2 \sigma(I)$

$R_{\text {int }}=0.036$

$\theta_{\text {max }}=26.0^{\circ}, \theta_{\min }=2.6^{\circ}$

$h=-5 \rightarrow 5$

$k=-19 \rightarrow 19$

$l=-16 \rightarrow 16$

\section{Refinement}

Refinement on $F^{2}$

Least-squares matrix: full

$R\left[F^{2}>2 \sigma\left(F^{2}\right)\right]=0.015$

$w R\left(F^{2}\right)=0.037$

$S=1.08$

1893 reflections

120 parameters

1 restraint

Hydrogen site location: inferred from

neighbouring sites

$\mathrm{H}$-atom parameters constrained

$w=1 /\left[\sigma^{2}\left(F_{\mathrm{o}}{ }^{2}\right)+(0.0115 P)^{2}+1.9359 P\right]$

where $P=\left(F_{\mathrm{o}}{ }^{2}+2 F_{\mathrm{c}}{ }^{2}\right) / 3$

$(\Delta / \sigma)_{\max }=0.001$

$\Delta \rho_{\max }=0.88$ e $\AA^{-3}$

$\Delta \rho_{\min }=-1.22 \mathrm{e} \AA^{-3}$

Special details

Geometry. All esds (except the esd in the dihedral angle between two 1.s. planes) are estimated using the full covariance matrix. The cell esds are taken into account individually in the estimation of esds in distances, angles and torsion angles; correlations between esds in cell parameters are only used when they are defined by crystal symmetry. An approximate (isotropic) treatment of cell esds is used for estimating esds involving l.s. planes. 
Fractional atomic coordinates and isotropic or equivalent isotropic displacement parameters $\left(\AA^{2}\right)$

\begin{tabular}{|c|c|c|c|c|}
\hline & $x$ & $y$ & $z$ & $U_{\text {iso }} * / U_{\text {eq }}$ \\
\hline $\mathrm{Pb} 1$ & $0.74423(2)$ & $0.09596(2)$ & $0.12971(2)$ & $0.01076(6)$ \\
\hline $\mathrm{O} 2$ & $1.0207(5)$ & $0.22282(15)$ & $0.19960(18)$ & $0.0179(5)$ \\
\hline $\mathrm{O} 1$ & $0.6719(5)$ & $0.24845(16)$ & 0.08699 (19) & $0.0185(5)$ \\
\hline $\mathrm{C} 1$ & $0.8763(7)$ & $0.2736(2)$ & $0.1450(2)$ & $0.0148(7)$ \\
\hline $\mathrm{C} 2$ & $0.9506(9)$ & $0.3666(2)$ & $0.1503(3)$ & $0.0272(8)$ \\
\hline $\mathrm{H} 2 \mathrm{~A}$ & 0.8392 & 0.3967 & 0.1002 & $0.041 *$ \\
\hline $\mathrm{H} 2 \mathrm{~B}$ & 1.1601 & 0.3743 & 0.1406 & $0.041 *$ \\
\hline $\mathrm{H} 2 \mathrm{C}$ & 0.9001 & 0.3884 & 0.2135 & $0.041 *$ \\
\hline $\mathrm{S} 1$ & $1.22553(19)$ & $0.09283(5)$ & $-0.02631(6)$ & $0.01392(17)$ \\
\hline $\mathrm{C} 3$ & $1.3066(7)$ & $0.0675(2)$ & $0.2991(3)$ & $0.0156(7)$ \\
\hline $\mathrm{O} 3$ & $1.2009(5)$ & $0.02818(15)$ & $0.22544(17)$ & $0.0166(5)$ \\
\hline $\mathrm{O} 4$ & $0.5375(6)$ & $0.11365(17)$ & 0.29615 (19) & $0.0204(5)$ \\
\hline $\mathrm{C} 5$ & $1.1245(7)$ & $0.1822(2)$ & -0.0929 & 0.0127 (6) \\
\hline N2 & $1.2377(6)$ & $0.25730(18)$ & -0.0723 & $0.0154(6)$ \\
\hline $\mathrm{H} 2 \mathrm{NB}$ & 1.1832 & 0.3011 & -0.1061 & $0.019 *$ \\
\hline H2NA & 1.3665 & 0.2628 & -0.0248 & $0.019 *$ \\
\hline N1 & $0.9253(6)$ & $0.1734(2)$ & $-0.1662(2)$ & $0.0194(6)$ \\
\hline H1NA & 0.8702 & 0.2170 & -0.2002 & $0.023 *$ \\
\hline H1NB & 0.8516 & 0.1240 & -0.1795 & $0.023 *$ \\
\hline $\mathrm{C} 4$ & $1.1663(9)$ & $0.0596(3)$ & 0.3979 (3) & $0.0280(8)$ \\
\hline $\mathrm{H} 4 \mathrm{~A}$ & 0.9607 & 0.0437 & 0.3892 & $0.042 *$ \\
\hline $\mathrm{H} 4 \mathrm{~B}$ & 1.1793 & 0.1132 & 0.4316 & $0.042 *$ \\
\hline $\mathrm{H} 4 \mathrm{C}$ & 1.2695 & 0.0168 & 0.4359 & $0.042 *$ \\
\hline
\end{tabular}

Atomic displacement parameters $\left(\AA^{2}\right)$

\begin{tabular}{lllllll}
\hline & $U^{11}$ & $U^{22}$ & $U^{33}$ & $U^{12}$ & $U^{13}$ & $U^{23}$ \\
\hline $\mathrm{Pb} 1$ & $0.01374(8)$ & $0.00827(8)$ & $0.01006(8)$ & $-0.00053(4)$ & $-0.00361(5)$ & $-0.00080(4)$ \\
$\mathrm{O} 2$ & $0.0227(12)$ & $0.0117(12)$ & $0.0188(13)$ & $-0.0023(9)$ & $-0.0098(10)$ & $0.0009(10)$ \\
$\mathrm{O} 1$ & $0.0213(12)$ & $0.0146(12)$ & $0.0191(13)$ & $-0.0020(9)$ & $-0.0092(10)$ & $-0.0023(10)$ \\
$\mathrm{C} 1$ & $0.0189(16)$ & $0.0118(16)$ & $0.0136(16)$ & $-0.0018(13)$ & $-0.0015(12)$ & $-0.0016(13)$ \\
$\mathrm{C} 2$ & $0.039(2)$ & $0.0124(18)$ & $0.029(2)$ & $-0.0067(15)$ & $-0.0150(17)$ & $0.0051(15)$ \\
$\mathrm{S} 1$ & $0.0199(4)$ & $0.0078(4)$ & $0.0137(4)$ & $-0.0009(3)$ & $-0.0069(3)$ & $0.0015(3)$ \\
$\mathrm{C} 3$ & $0.0180(16)$ & $0.0138(16)$ & $0.0146(17)$ & $0.0055(13)$ & $-0.0061(13)$ & $0.0008(13)$ \\
$\mathrm{O} 3$ & $0.0219(12)$ & $0.0127(12)$ & $0.0147(12)$ & $0.0018(9)$ & $-0.0084(9)$ & $-0.0010(9)$ \\
$\mathrm{O} 4$ & $0.0223(13)$ & $0.0199(12)$ & $0.0188(13)$ & $-0.0024(10)$ & $-0.0045(10)$ & $-0.0074(10)$ \\
$\mathrm{C} 5$ & $0.0152(14)$ & $0.0142(16)$ & $0.0087(15)$ & $0.0019(12)$ & $-0.0009(11)$ & $0.0032(12)$ \\
$\mathrm{N} 2$ & $0.0213(14)$ & $0.0115(14)$ & $0.0130(14)$ & $-0.0018(11)$ & $-0.0078(11)$ & $0.0046(11)$ \\
$\mathrm{N} 1$ & $0.0225(14)$ & $0.0173(15)$ & $0.0180(15)$ & $-0.0014(12)$ & $-0.0086(11)$ & $0.0063(12)$ \\
$\mathrm{C} 4$ & $0.0267(19)$ & $0.033(2)$ & $0.024(2)$ & $0.0037(16)$ & $-0.0017(16)$ & $0.0015(17)$ \\
& & & & & & \\
\hline
\end{tabular}

Geometric parameters $\left(\AA,{ }^{o}\right)$

\begin{tabular}{llll}
\hline $\mathrm{Pb} 1-\mathrm{O} 1$ & $2.483(2)$ & $\mathrm{S} 1-\mathrm{Pb} 1^{\mathrm{ii}}$ & $3.1121(9)$ \\
$\mathrm{Pb} 1-\mathrm{O} 4$ & $2.489(3)$ & $\mathrm{C} 3-\mathrm{O} 3$ & $1.261(4)$
\end{tabular}




\begin{tabular}{|c|c|c|c|}
\hline $\mathrm{Pb} 1-\mathrm{O} 2$ & $2.520(2)$ & $\mathrm{C} 3-\mathrm{O} 4^{\mathrm{ii}}$ & $1.265(4)$ \\
\hline $\mathrm{Pb} 1-\mathrm{O} 3$ & $2.626(2)$ & $\mathrm{C} 3-\mathrm{C} 4$ & $1.507(5)$ \\
\hline $\mathrm{Pb} 1-\mathrm{C} 1$ & $2.858(3)$ & $\mathrm{O} 4-\mathrm{C} 3^{\mathrm{i}}$ & $1.265(4)$ \\
\hline $\mathrm{Pb} 1-\mathrm{S} 1$ & $3.0701(9)$ & $\mathrm{C} 5-\mathrm{N} 2$ & $1.311(4)$ \\
\hline $\mathrm{Pb} 1-\mathrm{S} 1^{\mathrm{i}}$ & $3.1121(9)$ & $\mathrm{C} 5-\mathrm{N} 1$ & $1.330(4)$ \\
\hline $\mathrm{O} 2-\mathrm{C} 1$ & $1.259(4)$ & $\mathrm{N} 2-\mathrm{H} 2 \mathrm{NB}$ & 0.8600 \\
\hline $\mathrm{O} 1-\mathrm{C} 1$ & $1.259(4)$ & $\mathrm{N} 2-\mathrm{H} 2 \mathrm{NA}$ & 0.8600 \\
\hline $\mathrm{C} 1-\mathrm{C} 2$ & $1.499(5)$ & $\mathrm{N} 1-\mathrm{H} 1 \mathrm{NA}$ & 0.8600 \\
\hline $\mathrm{C} 2-\mathrm{H} 2 \mathrm{~A}$ & 0.9600 & $\mathrm{~N} 1-\mathrm{H} 1 \mathrm{NB}$ & 0.8600 \\
\hline $\mathrm{C} 2-\mathrm{H} 2 \mathrm{~B}$ & 0.9600 & $\mathrm{C} 4-\mathrm{H} 4 \mathrm{~A}$ & 0.9600 \\
\hline $\mathrm{C} 2-\mathrm{H} 2 \mathrm{C}$ & 0.9600 & $\mathrm{C} 4-\mathrm{H} 4 \mathrm{~B}$ & 0.9600 \\
\hline $\mathrm{S} 1-\mathrm{C} 5$ & $1.726(3)$ & $\mathrm{C} 4-\mathrm{H} 4 \mathrm{C}$ & 0.9600 \\
\hline $\mathrm{O} 1-\mathrm{Pb} 1-\mathrm{O} 4$ & $93.25(8)$ & $\mathrm{C} 1-\mathrm{C} 2-\mathrm{H} 2 \mathrm{C}$ & 109.5 \\
\hline $\mathrm{O} 1-\mathrm{Pb} 1-\mathrm{O} 2$ & $52.18(8)$ & $\mathrm{H} 2 \mathrm{~A}-\mathrm{C} 2-\mathrm{H} 2 \mathrm{C}$ & 109.5 \\
\hline $\mathrm{O} 4-\mathrm{Pb} 1-\mathrm{O} 2$ & $76.03(8)$ & $\mathrm{H} 2 \mathrm{~B}-\mathrm{C} 2-\mathrm{H} 2 \mathrm{C}$ & 109.5 \\
\hline $\mathrm{O} 1-\mathrm{Pb} 1-\mathrm{O} 3$ & $127.07(8)$ & $\mathrm{C} 5-\mathrm{S} 1-\mathrm{Pb} 1$ & $99.88(10)$ \\
\hline $\mathrm{O} 4-\mathrm{Pb} 1-\mathrm{O} 3$ & $84.07(8)$ & $\mathrm{C} 5-\mathrm{S} 1-\mathrm{Pb} 1^{\mathrm{ii}}$ & $121.89(12)$ \\
\hline $\mathrm{O} 2-\mathrm{Pb} 1-\mathrm{O} 3$ & $76.25(7)$ & $\mathrm{Pb} 1-\mathrm{S} 1-\mathrm{Pb} 1^{\mathrm{ii}}$ & $93.05(2)$ \\
\hline $\mathrm{O} 1-\mathrm{Pb} 1-\mathrm{S} 1$ & $86.73(6)$ & $\mathrm{O} 3-\mathrm{C} 3-\mathrm{O} 4^{\mathrm{ii}}$ & $123.1(3)$ \\
\hline $\mathrm{O} 4-\mathrm{Pb} 1-\mathrm{S} 1$ & $156.73(6)$ & $\mathrm{O} 3-\mathrm{C} 3-\mathrm{C} 4$ & $120.9(3)$ \\
\hline $\mathrm{O} 2-\mathrm{Pb} 1-\mathrm{S} 1$ & $85.78(6)$ & $\mathrm{O} 4 \stackrel{\mathrm{ii}}{-\mathrm{C} 3}-\mathrm{C} 4$ & $115.9(3)$ \\
\hline $\mathrm{O} 3-\mathrm{Pb} 1-\mathrm{S} 1$ & $77.53(5)$ & $\mathrm{C} 3-\mathrm{O} 3-\mathrm{Pb} 1$ & $118.0(2)$ \\
\hline $\mathrm{C} 1-\mathrm{Pb} 1-\mathrm{S} 1$ & $85.29(7)$ & $\mathrm{C} 3{ }^{\mathrm{i}}-\mathrm{O} 4-\mathrm{Pb} 1$ & $106.9(2)$ \\
\hline $\mathrm{O} 1-\mathrm{Pb} 1-\mathrm{S}^{\mathrm{i}}$ & $76.36(6)$ & $\mathrm{N} 2-\mathrm{C} 5-\mathrm{N} 1$ & $120.3(3)$ \\
\hline $\mathrm{O} 4-\mathrm{Pb} 1-\mathrm{S} 1^{\mathrm{i}}$ & $109.57(6)$ & $\mathrm{N} 2-\mathrm{C} 5-\mathrm{S} 1$ & $121.5(3)$ \\
\hline $\mathrm{O} 2-\mathrm{Pb} 1-\mathrm{S}^{\mathrm{i}}$ & $128.52(6)$ & $\mathrm{N} 1-\mathrm{C} 5-\mathrm{S} 1$ & $118.2(3)$ \\
\hline $\mathrm{O} 3-\mathrm{Pb} 1-\mathrm{S} 1^{\mathrm{i}}$ & $153.29(5)$ & $\mathrm{C} 5-\mathrm{N} 2-\mathrm{H} 2 \mathrm{NB}$ & 120.0 \\
\hline $\mathrm{S} 1-\mathrm{Pb} 1-\mathrm{S} 1^{\mathrm{i}}$ & $93.05(2)$ & $\mathrm{C} 5-\mathrm{N} 2-\mathrm{H} 2 \mathrm{NA}$ & 120.0 \\
\hline $\mathrm{C} 1-\mathrm{O} 2-\mathrm{Pb} 1$ & $92.08(19)$ & $\mathrm{H} 2 \mathrm{NB}-\mathrm{N} 2-\mathrm{H} 2 \mathrm{NA}$ & 120.0 \\
\hline $\mathrm{C} 1-\mathrm{O} 1-\mathrm{Pb} 1$ & $93.8(2)$ & $\mathrm{C} 5-\mathrm{N} 1-\mathrm{H} 1 \mathrm{NA}$ & 120.0 \\
\hline $\mathrm{O} 2-\mathrm{C} 1-\mathrm{O} 1$ & $121.9(3)$ & $\mathrm{C} 5-\mathrm{N} 1-\mathrm{H} 1 \mathrm{NB}$ & 120.0 \\
\hline $\mathrm{O} 2-\mathrm{C} 1-\mathrm{C} 2$ & $118.6(3)$ & $\mathrm{H} 1 \mathrm{NA}-\mathrm{N} 1-\mathrm{H} 1 \mathrm{NB}$ & 120.0 \\
\hline $\mathrm{O} 1-\mathrm{C} 1-\mathrm{C} 2$ & $119.6(3)$ & $\mathrm{C} 3-\mathrm{C} 4-\mathrm{H} 4 \mathrm{~A}$ & 109.5 \\
\hline $\mathrm{O} 2-\mathrm{C} 1-\mathrm{Pb} 1$ & $61.80(17)$ & $\mathrm{C} 3-\mathrm{C} 4-\mathrm{H} 4 \mathrm{~B}$ & 109.5 \\
\hline $\mathrm{O} 1-\mathrm{C} 1-\mathrm{Pb} 1$ & $60.11(17)$ & $\mathrm{H} 4 \mathrm{~A}-\mathrm{C} 4-\mathrm{H} 4 \mathrm{~B}$ & 109.5 \\
\hline $\mathrm{C} 2-\mathrm{C} 1-\mathrm{Pb} 1$ & $178.3(3)$ & $\mathrm{C} 3-\mathrm{C} 4-\mathrm{H} 4 \mathrm{C}$ & 109.5 \\
\hline $\mathrm{C} 1-\mathrm{C} 2-\mathrm{H} 2 \mathrm{~A}$ & 109.5 & $\mathrm{H} 4 \mathrm{~A}-\mathrm{C} 4-\mathrm{H} 4 \mathrm{C}$ & 109.5 \\
\hline $\mathrm{C} 1-\mathrm{C} 2-\mathrm{H} 2 \mathrm{~B}$ & 109.5 & $\mathrm{H} 4 \mathrm{~B}-\mathrm{C} 4-\mathrm{H} 4 \mathrm{C}$ & 109.5 \\
\hline $\mathrm{H} 2 \mathrm{~A}-\mathrm{C} 2-\mathrm{H} 2 \mathrm{~B}$ & 109.5 & & \\
\hline
\end{tabular}

Symmetry codes: (i) $x-1, y, z$; (ii) $x+1, y, z$.

Hydrogen-bond geometry $\left(\AA,{ }^{\circ}\right)$

\begin{tabular}{lllll}
\hline$D-\mathrm{H} \cdots A$ & $D-\mathrm{H}$ & $\mathrm{H} \cdots A$ & $D \cdots A$ & $D-\mathrm{H} \cdots A$ \\
\hline $\mathrm{N} 2-\mathrm{H} 2 N B \cdots \mathrm{O} 4^{\mathrm{iii}}$ & 0.86 & 1.99 & $2.836(4)$ & 169 \\
$\mathrm{~N} 2-\mathrm{H} 2 N A \cdots \mathrm{O} 1^{\mathrm{ii}}$ & 0.86 & 2.04 & $2.883(4)$ & 168
\end{tabular}




\section{data reports}

$\begin{array}{lllll}\mathrm{N} 1-\mathrm{H} 1 N A \cdots \mathrm{O} 2^{\mathrm{iv}} & 0.86 & 2.26 & 3.021(4) & 148 \\ \mathrm{~N} 1-\mathrm{H} 1 N B \cdots \mathrm{O} 3^{\mathrm{v}} & 0.86 & 2.48 & 3.311(4) & 163 \\ \mathrm{C} 4-\mathrm{H} 4 A \cdots \mathrm{O} 4 & 0.96 & 2.51 & 3.225(5) & 131\end{array}$

Symmetry codes: (ii) $x+1, y, z$; (iii) $x+1 / 2,-y+1 / 2, z-1 / 2$; (iv) $x-1 / 2,-y+1 / 2, z-1 / 2$; (v) $-x+2,-y,-z$. 